\title{
REMARKS ON PRESCRIBING GAUSS CURVATURE
}

\author{
XINGWANG XU AND PAUL C. YANG
}

\begin{abstract}
We study the nonlinear partial differential equation for the problem of prescribing Gauss curvature $K$ on $S^{2}$. We give an example of a rotationally symmetric $K$ for which the Kazdan-Warner obstruction is satisfied but the equation has no rotationally symmetric solution. On the other hand, we give a simple sufficient condition for solvability of the equation when $K$ is rotationally symmetric. Finally we give a sufficient condition for solvability when $K$ is not necessarily rotationally symmetric.
\end{abstract}

\section{INTRODUCTION}

The standard 2-sphere $S^{2}=\left\{x_{1}^{2}+x_{2}^{2}+x_{3}^{2}=1\right\}$ with its natural metric $g_{0}=d x_{1}^{2}+d x_{2}^{2}+d x_{3}^{2}$ has constant Gauss curvature $K_{0}=1$. Under a conformal change of metric $g=e^{2 u} g_{0}$, the Gauss curvature $K$ of the metric $g$ is given by the equation

$$
\Delta u+K e^{2 u}=1 .
$$

The problem of characterizing the function $K$ for which (1.1) has a solution has received much attention in the literature $[\mathrm{A}, \mathrm{BC}, \mathrm{Bo}, \mathrm{CY} 1, \mathrm{CY} 2, \mathrm{ChD}$, $\mathrm{ChS}, \mathrm{ES}, \mathrm{Ha}, \mathrm{Ho}, \mathrm{K}, \mathrm{KW}, \mathrm{M}, \mathrm{SZ}, \mathrm{Z}]$. In particular, we recall the subtle implicit condition discovered by Kazdan and Warner [KW]:

$$
\int\left\langle\nabla K, \nabla x_{k}\right\rangle e^{2 u}=0, \quad k=1,2,3 .
$$

This condition gives rise to many examples of $K$ for which (1.2) is not solvable. In particular, a rotationally symmetric function $K=K\left(x_{3}\right)$ which is monotone and nonconstant does not admit a solution. Although there has been many known criteria for the existence of solutions to equation (1.1) involving the nondegeneracy of the function $K$, the possibility that the following condition is a necessary and sufficient condition for the solvability of (1.1) remains open:

$(\alpha)$ There exists a function $u$ for which equation (1.2) holds.

In this article we will first give a family of rotationally symmetric functions $K_{\varepsilon}=K_{\varepsilon}\left(x_{3}\right)$ with the property that $K_{\varepsilon}^{\prime}$ changes sign, thus assuring the existence of function $u_{\varepsilon}$ satisfying (1.2). However, the family $K_{\varepsilon}$ has a monotone limit $K$ as $\varepsilon$ tends to 0 , thus the equation (1.1) does not have a solution. We will

Received by the editors October 30, 1990 and, in revised form, January 14, 1991.

1991 Mathematics Subject Classification. Primary 53C21, 58G03.

The second author's research was supported by NSF Grant 87-02871. 
show that for sufficiently small $\varepsilon$ the equation (1.1) with $K=K_{\varepsilon}$ cannot have a rotationally symmetric solution, by proving a compactness property of such a family of solutions $u_{\varepsilon}$.

In this example, the functions $K_{\varepsilon}$ have the property that $K_{\varepsilon}^{\prime}$ changes sign over the region where $K_{\varepsilon}$ is negative. It is natural to investigate the following condition:

$\left(\alpha^{\prime}\right) K^{\prime}$ has both positive and negative values in the region where $K>0$.

We will show that condition $\left(\alpha^{\prime}\right)$ is in fact sufficient condition for the solvability of equation (1.1) provided we impose a nondegeneracy condition on the critical points of $K$. More precisely, we prove

Theorem 2. Let $K=K\left(x_{3}\right)$ be a rotationally symmetric function which is nondegenerate, i.e., $K^{\prime \prime}(q) \neq 0$ where $K^{\prime}(q)=0$. If $K$ satisfies condition $\left(\alpha^{\prime}\right)$, then equation (1.1) has a solution.

Previously Hong [Ho] and Cheng-Smoller [ChS] had given sufficient conditions for the solvability of (1.1) as an ordinary differential equation. In the case of rotational symmetry an extremizing sequence of rotationally symmetric functions for the relevant functional can only blow up at the two poles. Hence, the nondegeneracy conditions are only imposed at the poles in the results of Hong and Cheng-Smoller. In our case we treat the equation as a partial differential equation and we need to impose nondegeneracy conditions at all the possible blowup points. We do not know whether the solutions we obtain are in fact rotationally symmetric.

As a finally remark we point out that in the general case where $K$ need not be rotationally symmetric, it suffices to consider the behavior of $\nabla K$ over the region where $K$ is positive. More precisely we have the following improvement of a previous result by one of us [CY2]:

Theorem 3. Let $K$ be a smooth function on $S^{2}$ with only nondegenerate critical points in the region $\Omega=\{p \mid K(p)>0\}$; in fact, we assume that $\Delta K \neq 0$ for all critical points $Q \in \Omega$ of $K$. Let $p+1=$ number of local maxima $Q \in \Omega$ of $K$, and $q=$ number of saddle points $Q \in \Omega$ with $\Delta K(Q)>0$.

If $p \neq q$, equation (1.1) has a solution.

The proof is a modification of the argument in [CY2].

The analysis required to prove Theorems 2 and 3 is the control of the concentration phenomenon as given in [CY1] and a companion compactness theorem for conformal metrics given in the appendix of [CY3].

\section{CONSEQuences of Moser's INEQuality}

In this section we recall without proof the basic analytic facts about concentrating mass distributions derived in [CY1] from the fundamental inequality of Moser [M].

For $u \in H^{1}, g=e^{2 u} g_{0}$ gives rise to a conformal metric on $S^{2}$. Under a conformal transformation of $S^{2}$ we denote the pull back metric $\phi^{*} g=e^{2 T[\phi](u)} g_{0}$, thus we have in particular, $T[\phi](u)=u \circ \phi+\frac{1}{2} \log (\operatorname{det} d \phi)$. We frequently make use of the following family of conformal transformations of $S^{2}$ : given $Q \in S^{2}$ and $t \geq 1$, let $y$ be the stereographic coordinates obtained by projecting from $Q$, as the North Pole, to the equatorial $y_{1}, y_{2}$ plane. $\phi_{Q, t}$ will denote the conformal transformation given in the $y$ coordinates by $\phi_{Q, t}(y)=t y$. The 
totality of such conformal transformations is homeomorphic to the unit ball in $R^{3}$, as we can make explicit by taking $q=\frac{t-1}{t} Q$. For $u \in H^{1}$, let

$$
S[u]=f|\nabla u|^{2}+2 u, \quad J[u]=\log f e^{2 u}-S[u], \quad F[u]=\log f K e^{2 u}-S[u] .
$$

The Euler equation for the critical point of the functional $F$ is given by (1.1).

Lemma 2.1 [O, OPS]. $J[u] \leq 0$, with equality only in the case $e^{2 u}=\operatorname{det} d \phi$, where $\phi$ is a conformal transformation of $S^{2}$.

Denote by $\mathscr{S}$ the class of functions $u \in H^{1}$ satisfying

$$
\int_{S^{2}} x_{i} e^{2 u} d v=0, \quad i=1,2,3 .
$$

Lemma 2.1' [CY1]. There exist a constant $1 / 2<a<1$ such that for $u \in \mathscr{S}$, we have

$$
f e^{2 u} \leq \exp \left[a f|\nabla u|^{2}+2 f u\right] .
$$

Lemma 2.2 (Concentration Lemma). Given a sequence $u_{j} \in H^{1}$ with $S\left[u_{j}\right] \leq C$, then either (i) $f|\nabla u|^{2} \leq C_{1}$ or (ii) a subsequence $e^{2 u_{j}} d A$ concentrates at a point $Q \in S^{2}:$ for any continuous function $f$ we have

$$
\lim _{j \rightarrow \infty} f f e^{2 u_{j}}=f(Q) .
$$

We introduce a parameter which measures the extent of concentration:

Definition. We say $u \in \mathscr{S}_{Q, t}$ if $T\left[\phi_{Q, t}\right](u) \in \mathscr{S}$. In this case, we also write $Q=Q(u)$ and $t=t(u)$. (Such a conformal transformation can be shown to exist by a simple topological argument.)

For very concentrated mass $e^{2 u} d A$, we have the following:

Lemma 2.3 (Asymptotic formula). For each $\mathscr{C}^{2}$ function $f$, we have

$$
\begin{aligned}
f f e^{2 u}= & f(Q)+2 \Delta f(Q) t^{-2}(\log t) \\
& +O\left(|\nabla f(Q)| t^{-1}(\log t)^{-1 / 2}(S[u])^{1 / 2}\right) .
\end{aligned}
$$

In order to apply the previous lemma effectively we need to make sure that the error term is of lower order than the main term. For this reason we introduce a flow in $H^{1}$ along which $S[u]$ decreases without changing the $(Q, t)$ parameter of concentration. We formulate this as the following

Lemma 2.4 (Lifting Lemma). Given $u_{0} \in \mathscr{S}_{Q, t}$ with $t$ large and $S[u] \leq \eta$, there exists a continuous path $u_{\gamma} \in H^{1}, 0 \leq \gamma \leq \gamma_{0}$, with $u(0)=u_{0}, u_{\gamma} \in \mathscr{S}_{Q, t}$, and $J\left[u_{\gamma}\right]$ and $F\left[u_{\gamma}\right]$ both increasing functions of $\gamma$ so that, at the endpoint,

$$
S\left[u_{\gamma_{0}}\right]=O(|\nabla K(Q)|)^{2} t^{-2} \log t+O\left(t^{-2}\right) .
$$

The Lifting Lemma was proved in [CY2] under the assumption that $K$ is strictly positive. Here we make the simple observation that in case $K$ is allowed to have negative values, the previous arguments still go through provided we 
start with $u_{0} \in H^{1 *}=\left\{u \in H^{1} \mid \int K e^{2 u} d v>0\right\}$. From this we observe that the flow introduced in [CY2] remains in the class $H^{1 *}$; namely, using

$$
f K e^{2 u_{\gamma}}=\exp \left\{F\left[u_{\gamma}\right]+S\left[u_{\gamma}\right]\right\}
$$

and the fact that $\frac{d}{d \gamma} F\left[u_{\gamma}\right]>0$ and $S\left[u_{\gamma}\right] \geq 0$, we see that $f K e^{2 u_{\gamma}}$ is always positive. Hence we have the (modified) Lifting Lemma 2.4.

\section{NONEXISTENCE}

Suppose $K_{\varepsilon}(\theta)$ is a family of smooth functions of $\theta$ satisfying the following condition: there exist $0<\delta_{0}<\delta_{1}<\delta_{2} \leq \delta_{3}<\pi$ such that

1. $K_{\varepsilon}\left(\delta_{0}\right)=0, K_{\varepsilon}\left(\delta_{1}\right)=-1, K_{\varepsilon}^{\prime}\left(\delta_{2}\right)=0, K_{\varepsilon}(0)=1,-2 \leq K_{\varepsilon} \leq+1$;

2. For $0<\theta<\delta_{3}, K_{\varepsilon}(\theta) \equiv K(\theta)$, i.e., $K_{\varepsilon}(\theta)$ does not depend on $\varepsilon$;

3. $K^{\prime}(\theta)<0$ for $0<\theta<\delta_{2}$ and $K^{\prime}(\theta) \leq 0$ for $\delta_{2} \leq \theta \leq \delta_{3}$;

4. $0 \leq K_{\varepsilon}^{\prime}(\theta)<\varepsilon$ for $\delta_{3} \leq \theta \leq \pi$;

5. $K(\theta)<-1$ for all $\delta_{1}<\theta \leq \pi$.

We set up coordinates $(\theta, \phi)$ on $S^{2}$ such that $0 \leq \theta \leq \pi, 0 \leq \phi \leq 2 \pi$, and $(0, \phi)=N,(\pi, \phi)=S$. We will prove the following:

Theorem 1. There exists an $\varepsilon_{0}>0$ such that $K_{\varepsilon}(\theta)$ cannot be realized as a Gaussian curvature of some metric on $S^{2}$ which is rotationally symmetric and pointwise conformal to the standard metric for all $\varepsilon \leq \varepsilon_{0}$.

We will use an indirect argument. Suppose the conclusion is false; then there exists a sequence $\varepsilon_{i}$ tending to zero such that the equation

$$
\Delta u+K_{\varepsilon_{i}} e^{2 u}=1
$$

would have a solution, $u_{\varepsilon_{i}}$. Integrating (3.1), we drop the subscript $i$ for convenience,

$$
\int_{S^{2}} K_{\varepsilon} e^{2 u_{\varepsilon}} d v=4 \pi
$$

Kazdan-Warner's condition [KW] applied to the rotationally symmetric eigenfunction $x_{3}=\cos (\theta)$ gives,

$$
\int_{0}^{2 \pi} \int_{0}^{\pi} K_{\varepsilon}^{\prime} \sin ^{2}(\theta) e^{2 u_{\varepsilon}} d \theta d \phi=0
$$

Lemma 3.1. The volume of $\left(S^{2}, e^{2 u_{\varepsilon}} g_{0}\right)$ is uniformly bounded from below and above.

Proof. Since $K_{\varepsilon} \leq 1$, by (3.2) we have

$$
4 \pi=\int_{S^{2}} K_{\varepsilon} e^{2 u_{\varepsilon}} d v \leq \int_{S^{2}} e^{2 u_{\varepsilon}} d v=\text { volume of }\left(S^{2}, e^{2 u_{\varepsilon}} g_{0}\right) .
$$

This proves the lower bound.

By Ahlfors's generalized Schwarz lemma, $u_{\varepsilon}$ is bounded from above by the hyperbolic metric on the disk $\delta_{1}<\theta \leq \pi$, and hence uniformly bounded from above on the smaller disk $\delta_{4} \leq \theta \leq \pi$, where $\delta_{1}<\delta_{4}<\delta_{2}$ is any fixed number. Therefore, we have

$$
\int_{0}^{2 \pi} \int_{\delta_{4}}^{\pi} e^{2 u_{\varepsilon}} \sin (\theta) d \theta d \phi \leq M
$$


Using (3.3), we have

$$
\begin{aligned}
0 & \leq \int_{0}^{2 \pi} \int_{\delta_{3}}^{\pi} K_{\varepsilon}^{\prime}[\sin (\theta)]^{2} e^{2 u_{\varepsilon}} d \theta d \phi \\
& <\varepsilon \int_{0}^{2 \pi} \int_{\delta_{3}}^{\pi} e^{2 u_{\varepsilon}} \sin (\theta) d \theta d \phi \leq \varepsilon M .
\end{aligned}
$$

Now using (3.3), (3.4), and the fact that $K^{\prime}(\theta) \leq 0$ on the interval $\left(0, \delta_{3}\right)$, we have

$$
\begin{aligned}
\int_{0}^{2 \pi} & \int_{\delta}^{\delta_{3}}\left[-K^{\prime}(\theta)\right][\sin (\theta)]^{2} e^{2 u_{\varepsilon}} d \theta d \phi \\
& \leq \int_{0}^{2 \pi} \int_{\delta_{3}}^{\pi} K^{\prime}(\theta) \sin ^{2}(\theta) e^{2 u_{\varepsilon}} d \theta d \phi \leq \varepsilon M
\end{aligned}
$$

for any $0 \leq \delta \leq \delta_{0}$.

Since $K^{\prime}(\theta) \sin (\theta)$ is bounded away from zero on $\left(\delta, \delta_{4}\right)$, we must have

$$
\int_{0}^{2 \pi} \int_{\delta}^{\delta_{4}} e^{2 u_{\varepsilon}} \sin (\theta) d \theta d \phi \leq M_{1}
$$

uniformly in $\varepsilon$, where $0<\delta<\delta_{0}$. We also have

$$
\begin{aligned}
\int_{0}^{2 \pi} & \int_{0}^{\delta} e^{2 u_{\varepsilon}} \sin (\theta) d \theta d \phi \\
& \leq\left(\inf _{0 \leq \theta \leq \delta} K\right)^{-1} \int_{0}^{2 \pi} \int_{0}^{\delta} K e^{2 u} \sin (\theta) d \theta d \phi \\
& \leq\left(\inf _{0 \leq \theta \leq \delta} K\right)^{-1}\left\{4 \pi-\int_{0}^{2 \pi} \int_{\delta}^{2 \pi} K e^{2 u} \sin (\theta) d \theta d \phi\right\} \leq C s t .
\end{aligned}
$$

Lemma 3.2. The diameter of $\left(S^{2}, e^{2 u_{\varepsilon}} g_{0}\right)$ is bounded.

Proof. By the Schwarz lemma we have a bound for the diameter of the region $\delta_{2} \leq \theta \leq \pi$. Since the metric is rotationally symmetric, by the method of the proof of the Rauch comparison theorem, we have a bound for distances in the $\theta$ direction on the region $0 \leq \theta \leq \delta$, hence for the diameter of the region $0 \leq \theta \leq \delta$ for $\delta<\delta_{0}$. Thus it suffices to give a bound for the diameter of the region $\delta \leq \theta \leq \delta_{2}$. This means that we need to bound the length of the radial geodesics:

$$
\int_{\delta}^{\delta_{2}} e^{u_{\varepsilon}}
$$

and the length of the $\phi$ circles $\theta=\theta_{0}$ for $\delta \leq \theta_{0} \leq \delta_{2}$ :

$$
2 \pi e^{u_{\varepsilon}\left(\theta_{0}\right)} \sin \left(\theta_{0}\right) \text {. }
$$

The length of the radial geodesic can be bounded using the Cauchy-Schwarz inequality:

$$
\int_{\delta}^{\delta_{2}} e^{u_{\varepsilon}} \leq\left[\int_{\delta}^{\delta_{2}} \frac{1}{\sin (\theta)} d \theta\right]^{1 / 2} \cdot\left(\int_{\delta \leq \theta \leq \delta_{2}} e^{2 u_{\varepsilon}} \sin (\theta) d \theta\right)^{1 / 2} \leq C s t
$$


To control the length of the $\phi$-circles we use the Fubini theorem. Let $\rho(\theta)=$ $\int_{0}^{\theta} e^{u_{\varepsilon}} d \theta$ be the radial distance from the $\phi$ circle to the North Pole, and let $A(\rho)=$ area of the ball of radius $\rho$ and $L(\rho)=$ length of the boundary circle $=$ $2 \pi \sin \theta e^{u_{\varepsilon}(\theta)}$. Differentiating, using Fubini's theorem, we have

$$
\frac{d A}{d \rho}=L(\rho)=2 \pi \sin (\theta) e^{u_{\varepsilon}(\theta)} .
$$

By the Bishop Comparison Theorem [BiC],

$$
\frac{d A / d \rho}{A} \leq \frac{d A_{0} / d \rho}{A_{0}} \text { for } 0 \leq \theta \leq \delta,
$$

where $A_{0}$ is the corresponding area function on the hyperbolic surface of curvature -2 . Since the radial diameter is bounded, we see that $\frac{d A_{0} / d \rho}{A_{0}}$ is a bounded function, and hence

$$
\frac{d A}{d \rho} \leq A M_{0} \leq(\sup A) M_{0}
$$

Lemma 3.3. $S\left[u_{\varepsilon}\right]$ is uniformly bounded from above.

Proof. By the previous argument and the Li-Yau estimates on the first eigenvalue [LY], we know that if $\lambda_{\varepsilon}$ is the first nonzero eigenvalue of the Laplace operator $\Delta_{\varepsilon}$ associated to the Riemannian metrics $e^{2 u_{\varepsilon}} g_{0}$ on $S^{2}$, then $\lambda_{\varepsilon} \geq$ $\Lambda>0$, where $\Lambda$ is a constant not depending on $\varepsilon$. By Lemma 3.1, we have

$$
\int_{S^{2}} K^{2} e^{2 u_{\varepsilon}} d v \leq M
$$

Then by [CY3, Theorem in Appendix], we see that there exists a conformal transformation $\phi_{\varepsilon}$ such that if we define

$$
w_{\varepsilon}=u_{\varepsilon} \circ \phi_{\varepsilon}+\frac{1}{2} \log \left|d \phi_{\varepsilon}\right|,
$$

then $\int_{S^{2}}\left|\nabla w_{\varepsilon}\right|^{2} d v$ is uniformly bounded from above. Consequently, $\left|\int_{S^{2}} w_{\varepsilon} d v\right|$ is also uniformly bounded from above. Therefore $S\left[w_{\varepsilon}\right]$ is uniformly bounded from above and below. It is well known that $S\left[w_{\varepsilon}\right]=S\left[u_{\varepsilon}\right]$. The proof of Lemma 3.3 is complete.

From the bounds on $\int\left|\nabla w_{\varepsilon}\right|^{2}$, we can derive immediately, using the Green's function, the following estimate:

$$
\begin{aligned}
\left|w_{\varepsilon}(Q)-f w_{\varepsilon}\right| & \leq\left|\int\left(-\Delta w_{\varepsilon}\right)(x) G(x, Q)\right| \leq \int\left|K_{\varepsilon} e^{2 w_{\varepsilon}}-1\right||G(x, Q)| d x \\
& \leq \sup \left|K_{\varepsilon}\right| \cdot\left(\int e^{4 w_{\varepsilon}}\right)^{1 / 2} \cdot\left(\int|G(x, Q)|^{2}\right)^{1 / 2}+C s t .
\end{aligned}
$$

Using Lemma 2.1 with $u=2 w_{\varepsilon}$,

$$
\int e^{4 w_{\varepsilon}} \leq \exp \left\{4 f\left|\nabla w_{\varepsilon}\right|^{2}+4 f w_{\varepsilon}\right\} \leq C s t .
$$

We see that $w_{\varepsilon}$ is bounded uniformly independent of $\varepsilon$. Rewriting (3.12) as $u_{\varepsilon}=w_{\varepsilon} \circ \phi^{-1}-\frac{1}{2} \log \left|d \phi_{\varepsilon}\right|$, we conclude that $u_{\varepsilon}$ is a bounded perturbation of the main term $-\frac{1}{2} \log \left|d \phi_{\varepsilon}\right|$. If $\phi_{\varepsilon}$ is a bounded sequence of conformal 
transformations, then a subsequence of $u_{\varepsilon}$ converges to a function and we can pass to the limit to obtain a function $u$ satisfying

$$
\Delta u+\left(\lim _{\varepsilon \rightarrow 0} K_{\varepsilon}\right) e^{2 u}=1 .
$$

This contradicts the Kazdan-Warner condition. On the other hand, if $\phi_{\varepsilon}$ is not a bounded sequence of conformal transformations, say $\phi_{\varepsilon_{j}}=\phi_{Q_{j}, t_{j}}$ with $t_{j}$ tending to $\infty$, it follows that a subsequence $Q_{j}$ converges to some $Q \in S^{2}$. We must have $\theta(Q) \leq \delta_{1}$, for otherwise $e^{2 u_{\varepsilon_{j}}}$ would be unbounded in contradiction to the Schwarz lemma. On the other hand, we have

$$
\lim _{j \rightarrow \infty} \int\langle\nabla K, \nabla \cos (\theta)\rangle e^{2 u_{\varepsilon}}=-K^{\prime}(\theta(Q)) \sin (\theta(Q))>0,
$$

again in contradiction with the Kazdan-Warner condition if $Q$ is not the North Pole. Finally in the case $Q$ is the North Pole, we can evaluate asymptotically the integral, using $\alpha=K^{\prime \prime}(0)<0$,

$$
\int_{0 \leq \theta \leq \delta_{3}}\langle\nabla K, \nabla \cos (\theta)\rangle e^{2 u_{\varepsilon}}=\alpha \frac{1}{t_{j}^{2}} \log t_{j}+O\left(\frac{1}{t_{j}^{2}}\right),
$$

while

$$
\int_{\delta_{3} \leq \theta \leq \pi}\langle\nabla K, \nabla \cos (\theta)\rangle e^{2 u_{\varepsilon}}=O\left(\frac{1}{t_{j}^{2}}\right) .
$$

This again gives a contradiction to the Kazdan-Warner condition.

\section{EXISTENCE RESULTS}

We give the proofs of Theorems 2 and 3 in this section.

Proof of Theorem 2. We introduce a 2-dimensional variational scheme. Let the local positive maxima of $K$ be $\theta_{1}, \theta_{2}, \ldots, \theta_{N}$ listed in the order $0<K\left(\theta_{1}\right) \leq$ $K\left(\theta_{2}\right) \leq \cdots \leq K\left(\theta_{N}\right)$. These correspond in general to circles $\theta=\theta_{i}$ on the sphere, except when $\theta_{i}$ is at the end point. Fix a plane domain $\Omega$ obtained by deleting $N-1$ disjoint disks from a large disk. Label the boundary circles of $\Omega$ as $\Gamma_{1}, \Gamma_{2}, \ldots, \Gamma_{N}$. Fix a diffeomorphism $\varphi$ mapping each $\Gamma_{i}$ onto the circle $\theta=\theta_{i}$, and in the case $\theta=\theta_{i}$ is a pole, map all of $\Gamma_{i}$ to the pole. Let $\mathscr{T}$ be the family of continuous maps $u: \Omega \mapsto H^{1 *}\left(S^{2}\right)$ with the properties

$$
\lim _{p \rightarrow \partial \Omega} S\left[u_{p}\right]=0
$$

and

$$
\lim _{p \rightarrow \zeta \in \partial \Omega} e^{2 u_{p}} d A=\delta_{\varphi(\zeta)},
$$

where $H^{1 *}=\left\{v \in H^{1} \mid \int K e^{2 v}>0\right\}$.

The max-min problem we consider is the following: Let

$$
\mu=\sup _{u \in \mathscr{T}} \min _{p \in \Omega} F\left[u_{p}\right]
$$

Claim 1. For each $u \in \mathscr{T}$, the minimum is achieved at some $p \in \Omega$ and this minimum value $F\left[u_{p}\right]$ satisfies

$$
F\left[u_{p}\right] \leq \log K\left(\theta_{1}\right)-\varepsilon .
$$


Proof of Claim 1. It follows from assumption $\left(\alpha^{\prime}\right)$ that either $N=1$ in which case $\theta=\theta_{1}$ is not a pole, or $N>1$ so that there exist at least two boundary curves in $\Gamma_{1}, \Gamma_{2}, \ldots, \Gamma_{N}$. Consider the center of mass surface $S: p \mapsto \int e^{2 u_{p}} \vec{X} / \int e^{2 u_{p}}=C . M .\left(e^{2 u_{p}}\right)$. Let $T_{\varepsilon}$ be the tubular neighborhood of the curve $\left\{\theta=\theta_{1}\right\}$. By the observation above, $\partial\left(T_{\varepsilon}\right)$ must meet $S$ at some point $\int e^{2 u_{p}} \vec{X} / \int e^{2 u_{p}}$. When $\varepsilon$ is sufficiently small, $e^{2 u_{p}} d A$ is very concentrated. Thus we can apply the asymptotic formula to see that

$$
F\left[u_{p}\right]=\log \left(\frac{1}{4 \pi} \int K e^{2 u_{p}} d A\right)-S\left[u_{p}\right]<\log K\left(\theta_{1}\right)-\varepsilon .
$$

We consider a maximizing sequence $u_{p_{l}}^{(i)}$ of minima, satisfying $F\left[u_{p}^{(i)}\right] \geq$ $F\left[u_{p_{i}}^{(i)}\right], F\left[u_{p_{i}}^{(i)}\right] / \mu$. We need to show that a subsequence of $u_{p_{i}}^{(i)}$ converges. If the Dirichlet integrals $\int\left|\nabla u_{p_{i}}^{(i)}\right|^{2}$ remain bounded, then a subsequence would converge to a weak limit which is in fact a solution of equation (1.1).

Suppose for the maximizing sequence $u_{p_{i}}^{(i)}$, there is no upper bound for $\int\left|\nabla u_{p_{i}}^{(i)}\right|^{2}$. From the inequality

$$
\mu-\varepsilon \leq F\left[u_{p_{i}}^{(i)}\right]=\log \left(\frac{1}{4 \pi} \int K e^{2 u_{p_{i}}^{(i)}}\right)-S\left[u_{p_{i}}^{(i)}\right],
$$

we see that $S\left[u_{p_{i}}^{(i)}\right] \leq C s t$. Thus the Concentration Lemma shows that a subsequence of $u_{p_{i}}^{(i)}$ concentrates at a point $Q_{0}$. Clearly we must have $K\left(Q_{0}\right)>0$. The Lifting Lemma then shows that we may assume without loss of generality that $S\left[u_{p_{i}}^{(i)}\right] \ll 1$ when $i$ is sufficiently large.

Claim 2. $\theta\left(Q_{0}\right)$ is a critical point of $K$.

Proof of Claim 2. Assume on the contrary that $\nabla K\left(\theta\left(Q_{0}\right)\right) \neq 0$. Let $T_{\varepsilon}$ be a tubular neighborhood of the circle $\theta=\theta\left(Q_{0}\right)$. We will modify the maps $u_{p}^{(i)}$ over the portion $\Delta=\left\{p \in \Omega \mid u_{p}^{(i)} \in S_{Q, t}\right.$ with $\left.q=(t-1) / t Q \in T_{\varepsilon}\right\}$, to obtain a competing map $v_{p}^{(i)}$ such that the $q$ parameter of $v_{p}^{(i)}$ no longer enters the region $T_{\varepsilon}$, but, $F\left[v_{p}^{(i)}\right] \geq F\left[u_{p}^{(i)}\right]$ for each $p \in \Delta$. Assume that $K^{\prime}\left(\theta\left(Q_{0}\right)\right)>0$. Let $\chi$ be a cut-off function: $\chi=1$ on $\theta\left(Q_{0}\right)-\varepsilon / 2 \leq \theta \leq \theta\left(Q_{0}\right)+\varepsilon / 2 ; \chi=0$ on $\theta<\theta\left(Q_{0}\right)-\varepsilon$ and $\theta>\theta\left(Q_{0}\right)+\varepsilon$; and otherwise varies linearly. Observe that from

$$
F\left[u_{p_{i}}^{(i)}\right]=\min _{p} F\left[u_{p}^{(i)}\right]
$$

we see that whenever the $Q$ parameter of $u_{p}^{(i)}$ lies outside the interval $\left(\theta\left(Q_{0}\right)-\right.$ $\left.\varepsilon, \theta\left(Q_{0}\right)+\varepsilon\right)$, the $t$ parameter must stay bounded, say $t \leq t_{0}$.

We will move the function $u$ in the following way: Let $\Psi_{s}$ be rotation by angle $s$ around the axis perpendicular to the plane spanned by $Q_{0}$ and $\nabla K\left(Q_{0}\right)$. Define

$$
v_{p}^{(i), s}= \begin{cases}u_{p}^{(i)} & \text { if } p \notin \Delta \\ T\left[\Psi_{s \chi\left(\theta\left(Q\left(u_{p}^{(i)}\right)\right)\right)}\right]\left(u_{p}^{(i)}\right) & \text { if } p \in \Delta .\end{cases}
$$


It is clear that $v_{p}^{(i), s} \in \mathscr{T}$ and that

$$
\begin{aligned}
\frac{d}{d s} F\left[v_{p}^{(i), s}\right] & =\frac{d}{d s} \log f K e^{2 v_{p}^{(i), s}}=\frac{d}{d s} \log f K \circ \Psi_{-s \chi\left(\theta\left(Q\left(u_{p}^{(i)}\right)\right)\right)} e^{2 u_{p}^{(i)}} \\
& =\left[f K e^{2 v_{p}^{(i)}}\right]^{-1} \cdot f\left\langle\nabla K, \frac{d}{d s} \Psi_{-s \chi\left(\theta\left(u_{p}^{(i)}\right)\right)}\right\rangle e^{2 u_{p}^{(i)}}>0
\end{aligned}
$$

by the asymptotic formula.

Observe that the effect of the transformation $T\left[\Psi_{s}\right]$ on the $(Q, t)$ parameter is given by $Q\left(T\left[\Psi_{s}\right]\right)(u)=\Psi_{s}(Q(u))$, while the $t$ parameter is unchanged.

Hence we may let $v_{p}^{(i)}=v_{p}^{(i), 1} . v_{p}^{(i)}$ is a better competing map whose minimum does not concentrate at $\theta=\theta\left(Q_{0}\right)$. This proves Claim 2 .

It follows from Claim 2 that $Q_{0}$ must be a local minimum.

Claim 3. $Q_{0}$ cannot be a local minimum.

Proof of Claim 3. Assume that $Q_{0}$ is a local minimum of $K$, i.e., $\theta_{0}=\theta\left(Q_{0}\right)$, $K^{\prime}\left(\theta_{0}\right)=0$, and $K^{\prime \prime}\left(\theta_{0}\right)>0$. We retain our notation as in the proof of Claim 2 , and we will modify $u^{(i)}$ over the region $\Delta$ to construct a competing map $v^{(i)}$ with the property that $F\left[v_{p}^{(i)}\right]=F\left[u_{p}^{(i)}\right]$ for all $p \in \Omega$, but that $v_{p}^{(i)}$ does not concentrate near $\theta=\theta_{0}$. More precisely, the $Q$ parameter of $v_{p}^{(i)}$ does not enter the region $T_{\varepsilon}$. Choose $\varepsilon$ sufficiently small so that for $\theta \in\left(\theta_{0}-\varepsilon, \theta_{0}+\varepsilon\right)$, we have $K^{\prime \prime}(\theta) \geq \delta>0$. We let $v_{p}^{(i), t}=u_{p}^{(i)}$ as before when $p \notin \Delta$. When $p \in \Delta$, let $(Q(p), t(p))$ denote the $(Q, t)$ parameter of the function $u_{p}^{(i)}$. Let $\chi$ be a cut-off function as before, and let $v_{p}^{(i), s}=T\left[\varphi_{Q\left(u^{(i)}\right), 1+s \chi\left(Q\left(u^{(i)}\right)\right)}\right]\left[u_{p}^{(i)}\right]$ for $p \in \Delta$. Then it is again clear that $v_{p}^{(i), t} \in \mathscr{T}$ and that

$$
\begin{aligned}
\frac{d}{d s} F\left[v_{p}^{(i), t}\right] & =\frac{d}{d t}\left[\log \frac{1}{4 \pi} \int K e^{2 v_{p}^{(i), t}}\right] \\
& =\frac{d}{d s} \log f K \circ \varphi_{Q\left(u^{(i)}\right), 1+s \chi\left(Q\left(u_{p}^{(i)}\right)\right)}^{-1} e^{2 u_{p}^{(i)}} \\
& =\left[\int K e^{2 v_{p}^{(i), s}}\right]^{-1} \int \chi\left\langle\nabla K, \nabla\left\langle\cdot, Q\left(u_{p}^{(i)}\right)\right\rangle\right\rangle e^{2 u_{p}^{(i)}}>0
\end{aligned}
$$

by the asymptotic formula. Observe that the effect of $T\left[\varphi_{Q\left(u_{p}^{(i)}\right), 1+s \chi\left(Q\left(u_{p}^{(i)}\right)\right)}\right]$, the transformation on the $(Q, t)$ parameter, is to leave the $Q$ parameter alone while reducing the $t$ parameter.

Hence we may let $v_{p}^{(i)}=v_{p}^{(i), 1}$. Then $v_{p}^{(i)}$ is a competing map with the stated property, and Claim 3 is proved.

This finishes the proof of Theorem 2.

Proof of Theorem 3. Here we simply observe that the same proof as given in [CY2] still works with the modification that all test maps take values in the space $H^{1 *}=\left\{u \in H^{1} \mid \int K e^{2 u}>0\right\}$. We argue that if a maximizing sequence of minima does not converge, then $u_{p}^{(i)}$ must concentrate at a point $Q_{0}$, where $K\left(Q_{0}\right)>0$. As we observed in $\S 2$, the Lifting Lemma still works in this case, thus we can proceed as before to show that at least one of the variational schemes must converge. 


\section{REFERENCES}

[A] T. Aubin, Meilleures constants dans le théorèmes d'inclusion de Sobolev et un théorème de Fredholm non linéaire pour la transformation conforme de la courbure scalaire, J. Funct. Anal. 32 (1979), 149-179.

[BC] A. Bahri and J. M. Coron, Une théorie des points critiques á l'infini pour l'equation de Yamabe et le probléme de Kazdan-Warner, C. R. Acad. Sci. Paris Sér. I 15 (1985), 513516.

[BiC] R. Bishop and R. Crittenden, Geometry of manifolds, Academic Press, New York, 1964.

[Bo] J. P. Bourguignon, Invariants integraux fonctionnels pour des equations aus derivees partielles d'origine geometrique, Lecture Notes in Math., vol. 1209, Springer, 1985, pp. 100-118.

[CY1] A. Chang and P. Yang, Prescribing Gaussian curvature on $S^{2}$, Acta Math. 159 (1987), 215-259.

[CY2] - Conformal deformation of metrics on $S^{2}$, J. Differential Geom. 27 (1988), 259-296.

[CY3] _- Isospectral conformal metrics on 3-manifolds, J. Amer. Math. Soc. 3 (1990), 117-145.

[CY4] _ A perturbation result in prescribing scalar curvature on $S^{n}$, preprint, 1990.

[ChD] W. X. Chen and W. Ding, Scalar curvature on $S^{2}$, Trans. Amer. Math. Soc. 303 (1987), 365-387.

[ChS] K.-S. Cheng and J. Smoller, On conformal metrics with prescribed Gaussian curvature on $S^{2}$, preprint, 1990.

[ES] J. Escobar and R. Schoen, Conformal metrics with prescribed scalar curvature, Invent. Math. 86 (1986), 243-254.

[Ha] Z.-C. Han, Ph.D. thesis, New York University, 1989.

[Ho] C. Hong, A best constant and the Gaussian curvature, Proc. Amer. Math. Soc. 97 (1986), 737-747.

[K] J. Kazdan, Ann. of Math. Stud., no. 102, Princeton Univ. Press, Princeton, N. J., 1982, pp. 185-191.

[KW] J. Kazdan and F. Warner, Curvature function for compact 2-manifold, Ann. of Math. (2) 99 (1974), 14-47.

[LY] P. Li and S.-T. Yau, Estimates of eigenvalues of a compact Riemannian manifold, Geometry of the Laplace Operator, Proc. Sympos. Pure Math., vol. 36, Amer. Math. Soc., Providence, R. I., 1980, pp. 205-239.

[M] J. Moser, On a non-linear problem in differential geometry, Dynamical Systems, Academic Press, New York, 1973.

[O] E. Onofri, On the positivity of the effective action in a theory of random surfaces, Comm. Math. Phys. 86 (1982), 321-326.

[OPS] B. Osgood, R. Phillips, and P. Sarnak, Extremals of determinants of Laplacians, J. Funct. Anal. 80 (1988), 148-211.

[SZ] R. Schoen and D. Zhang, private communication.

[Z] D. Zhang, Ph.D. Thesis, Stanford University, 1990.

Department of Mathematics, National University of Singapore, Lower Kent Ridge ROAD, SingaPORE 0511, SingaPORE

E-mail address: matxuxw@nusvm.bitnet

Department of Mathematics, University of Southern California, Los Angeles, CaliFORNIA 90089-1113

E-mail address: dyang@mtha.usc.edu 